\title{
Prenatal Pesticide and PCB Exposures and Birth Outcomes
}

\author{
MARY S. WOLFF, STEPHANIE ENGEL, GERTRUD BERKOWITZ, SUSAN TEITELBAUM, JODI SISKIND, DANA B. BARR, \\ AND JAMES WETMUR

\begin{abstract}
Department of Community and Preventive Medicine [M.S.W., S.E., G.B., S.T., J.S.], Department of Microbiology and Human Genetics [J.W.], Mount Sinai School of Medicine, New York, New York 10029; and National Center for Environmental Health [D.B.B.], Centers for Disease Control and Prevention, Atlanta, Georgia 30333
\end{abstract}

\begin{abstract}
Evidence is inconsistent or poorly understood for links between polychlorinated biphenyls (PCBs), 1, 1' -dichloro-2,2' bis(4-chlorophenyl)ethylene (DDE), and organophosphate (OP) pesticides and adverse pregnancy outcomes, although they are known developmental toxicants. We measured biomarkers of maternal exposure to DDE, PCB, and OP metabolites in the third trimester of pregnancy among 404 mothers in a multiethnic cohort in New York City. We also determined maternal paraoxonase (PON1), butyrylcholinesterase (BuChe), and PON1Q192R gene variant. Higher multivariate-adjusted DDE levels (but not $\mathrm{PCB}$ ) were associated with lower birth weight $\left(-98 \mathrm{~g} / \log _{10} \mathrm{DDE}, p=0.096\right)$ and head circumference $\left(-0.54 \mathrm{~cm} / \log _{10}\right.$ DDE, $\left.p=0.030\right)$. DDE and PCB levels were not related to birth length, Ponderal index, or gestational age. Birth length was shorter for mothers with PON192RR slow genotype compared with PON192QQ ( $p=0.026)$, and head circumference was inversely associated with maternal PON1 activity $(p=0.004)$. With slow-activity PON1 or PON192, urinary diethylphosphates ( $\Sigma$ DEPs) were associated with lower birth weight and dimethylphosphates ( $\Sigma$ DMPs) with shorter birth length. No associations were found between birth outcomes and BuChe. In summary, we found suggestive relationships between prenatal environmental biomarkers and birth outcomes in this population. Maternal susceptibility factors including PON1 and maternal weight contributed to the observed effects. (Pediatr Res 61: 243-250, 2007)
\end{abstract}

$\mathrm{T}$ here is widespread concern about potentially adverse health effects of environmental chemicals on children. Infants exposed in utero and during the early neonatal period are particularly vulnerable because of their rapid growth, cell differentiation, immaturity of metabolic pathways, and development of vital organ systems (1). Pesticides including persistent organochlorines (OCs) such as DDE and PCBs have been associated with detrimental effects on childhood neurobehavioral development (2), but evidence is inconsistent regarding their effects on birth outcomes. Furthermore, only recently have data on effects of nonpersistent pesticide exposure have begun to emerge (3-5). Because adverse birth outcomes may be intermediate between prenatal toxic exposures and child neurologic effects, the in utero effects of environmental agents on pregnancy outcomes are of interest.

Received May 12, 2006; accepted September 26, 2006

Correspondence: Mary S. Wolff, Ph.D., Department of Community and Preventive Medicine, Mount Sinai School of Medicine, One Gustave L. Levy Place, Box 1057, New York, NY 10029; e-mail: mary.wolff@mssm.edu

This research was supported by NIEHS/EPA Children's Center grants ES09584 and R827039, The New York Community Trust, and ATSDR/CDC/ATPM.

DOI: $10.1203 / p d r .0 b 013 e 31802 d 77 f 0$
OCs continue to be detected in women of childbearing age (6-8), although such chemicals were banned from commercial use in the 1970s. OPs have been widely used for decades and represent about half of insecticides used in the United States (9). Before 2000, approximately 60 million pounds were used annually in agriculture and another 17 million pounds for nonagricultural purposes, including spraying in residential buildings (9). OPs are highly neurotoxic, yet developmental effects in children have been little studied. Exposure has been documented for the general population including pregnant women and their children both in rural (10) and urban areas $(4,11)$ as well as in agricultural populations $(5)$.

The present investigation examines effects of in utero exposure to OPs and OCs on fetal growth in an urban cohort of mothers and their infants. We have previously reported results of effects on birth outcomes of exposure to 3,5,6-trichloro-2pyridinol, a metabolite of the OP pesticide chlorpyrifos as well as 3-phenoxybenzoic acid, a pyrethroid metabolite, and pentachlorophenol, a wood preservative and a metabolite of hexachlorobenzene (3). In this report, we describe birth outcomes with respect to OP metabolites, PCBs, DDE, and lead measured in maternal samples obtained in the third trimester of pregnancy.

\section{MATERIALS AND METHODS}

The Children's Environmental Health Study is a prospective study that is following an ethnically diverse cohort of mother-infant pairs enrolled at Mount Sinai Hospital in New York City during pregnancy from March 1998 to March 2002. Details regarding study design and protocols have been described previously (3). Excluded were multiparas; multiple gestations; first prenatal visit after 26 wk of gestation; serious chronic diseases such as diabetes, hypertension, and thyroid disease; serious pregnancy complication; consuming more than two alcoholic beverages per day; and using illegal drugs. Of 479 recruited women, 75 were excluded because of medical complications, severe prematurity ( $<1500 \mathrm{~g}$ or $<32 \mathrm{wk}$ of gestation), infant birth defects, no biologic specimens collected before birth, change of residence outside New York City, or refusal to continue participation. The final sample size for this analysis was 404 births. This study was approved by the Institutional Review Board of Mount Sinai School of Medicine, and signed informed consent was obtained.

Abbreviations: BuChe, butyrylcholinesterase; DDE, 1,1' ${ }^{\prime}$ dichloro-2,2' bis(4-chlorophenyl)ethylene; DEDP, diethyldithiophosphate; DMDP, dimethyldithiophosphate; DMTP, dimethylthiophosphate; LOD, limit of detection; MDA, malathion dicarboxylic acid; OCs, organochlorines; OP, organophosphate; PCBs, polychlorinated biphenyls; PON1, paraoxonase;

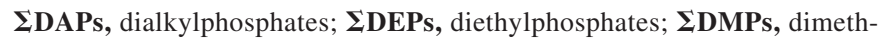
ylphosphates 
Table 1. Blood and urine analytes in prenatal maternal specimens

\begin{tabular}{|c|c|c|c|c|c|c|}
\hline & $n$ & LOD & $\%>$ LOD & Median & Min & Max \\
\hline \multicolumn{7}{|l|}{ Blood measures } \\
\hline Blood lead, $\mu \mathrm{g} / \mathrm{dL}$ & 292 & 0.1 & 88.0 & 0.90 & 0.10 & 6.3 \\
\hline $\mathrm{DDE}, \mu \mathrm{g} / \mathrm{L}$ & 194 & 0.07 & 97.4 & 0.64 & 0 & 57.3 \\
\hline DDE, ng/g lipid* & 174 & 10 & 98.9 & 110 & 0 & 9240 \\
\hline PCBs, ng/g lipid* & 174 & 50 & 94.8 & 150 & 20 & 1060 \\
\hline \multicolumn{7}{|l|}{ Urine measures } \\
\hline$\Sigma$ DAPs, $n m / L \dagger(\Sigma$ DAP $=\Sigma$ DMP $+\Sigma$ DEP $)$ & 327 & $\ddagger$ & 97.2 & 75.9 & 0 & 4987 \\
\hline$\Sigma$ DEPs, $\mathrm{nm} / \mathrm{g}$ creatinine & 327 & $\ddagger$ & 88.1 & 22.1 & 0 & 1002 \\
\hline$\Sigma$ DMPs, $\mathrm{nm} / \mathrm{g}$ creatinine & 336 & 末 & 92.6 & 55.4 & 0 & 2071 \\
\hline$\Sigma$ DAPs, $\mathrm{nm} / \mathrm{g}$ creatinine $(\Sigma \mathrm{DAP}=\Sigma \mathrm{DMP}+\Sigma \mathrm{DEP})$ & 327 & $\$$ & 97.2 & 88.6 & 0 & 2106 \\
\hline MDA $\mu \mathrm{g} / \mathrm{L} \dagger$ & 361 & 0.3 & 20.5 & LOD & LOD & 15.8 \\
\hline
\end{tabular}

* PCBs are the sum of four congeners, $118,138,153$, and 180. LOD is approximate based on the LOD $(\mu \mathrm{g} / \mathrm{L}) / \mathrm{mean}$ total lipids $(5.5 \mathrm{~g} / \mathrm{L})$; lipids were not available for 20 samples.

$\dagger$ Total number includes samples with creatinine $\geq 20$.

$\ddagger$ Based on an individual analyte value equal to or greater than LOD for any of the three metabolites in each class $(1-4 \mathrm{~nm} / \mathrm{L}, 0.2-0.5 \mu \mathrm{g} / \mathrm{L})$.

A questionnaire was administered to participants during their third trimester of pregnancy to obtain information on pesticide and other environmental exposures, sociodemographic characteristics, obstetric and medical history, and lifestyle factors. Information on delivery characteristics and birth outcomes, including birth weight, length, head circumference, gestational age, and infant gender were obtained from a computerized perinatal database within the Department of Obstetrics, Gynecology, and Reproductive Science at Mount Sinai Hospital. Measurements of weight, length, and head circumference at birth were based on standardized clinical techniques. Maternal blood and urine samples were obtained during the third trimester (generally between 26 and $28 \mathrm{wk}$ ). Cord blood samples were obtained at the time of birth. Maternal blood plasma was used to analyze DDE and PCB levels in a random subset of women $(n=194)$. Maternal urine samples collected before January $2001(n=386)$ were analyzed by the Centers for Disease Control and Prevention (CDC) for six alkylphosphate metabolites (APs) and malathion dicarboxylic acid (MDA). Lead levels $(\mathrm{PbB})$ were also measured in cord blood $(n=102)$ or maternal whole blood $(n=192)$ if a cord blood sample was not available.

Laboratory and quality control methods have been reported previously (7,12-14). Limits of detection (LOD) based on three times the standard deviation (SD) of blanks run during the determinations are presented in Table 1. Laboratory values were available for diethyldithiophosphate (DEDP) $(n=$ $344 ; 7 \%>$ LOD), LDEP $(n=316 ; 45 \%>$ LOD $)$, diethylthiophosphate $(n=$ $348 ; 81 \%>$ LOD), dimethyldithiophosphate (DMDP) $(n=357 ; 25 \%$ $>$ LOD), dimethylphosphate ( $\Sigma$ DMP) $(n=328 ; 58 \%>$ LOD $)$, dimethylthiophosphate (DMTP) $(n=346 ; 89 \%>$ LOD). Samples of urine with $<20$ $\mathrm{mg} / \mathrm{dL}$ creatinine $(n=27)$ were excluded from metabolite analyses. Concentrations were imputed for missing values (5); metabolites were then summed (as $\mathrm{nm} / \mathrm{L}$ ) to obtain $\Sigma$ DEP (DEDP, diethylphosphate, diethylthiophosphate),

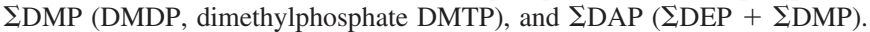
PON1 activity was determined as previously reported in plasma $(\mu \mathrm{mol} / \mathrm{min} /$ $\mathrm{mL})$ using phenylacetate as substrate $(15)$. BuChe activity $(\mu \mathrm{mol} / \mathrm{min} / \mathrm{mL})$ was determined by the method of Ellman et al. (16) using $1 \mathrm{mmol} / \mathrm{L}$ butyrylthiocholine as substrate in $50 \mathrm{mmol} / \mathrm{L}$ phosphate buffer, $\mathrm{pH} 8.0$, at $25^{\circ} \mathrm{C}$, following absorbance at $412 \mathrm{~nm}$. The assay within-batch precision was $3.2 \%$ for PON1 and $8.6 \%$ for BuChe. PONQ192R was genotyped $(n=389)$ as reported (17). This PON1 variant was chosen because its maternal phenotypic variability exhibits a range of PON1 expression different from other PON1 variants $(15,18)$. PCBs were defined as the sum of congeners 118,153 , 138, and 180 (7). Total lipids (g/L) were calculated using cholesterol and triglycerides (19) determined on 174 plasma samples with sufficient volume.

Statistical analyses were performed using SAS-PC, version 9.1 (SAS, Inc., Cary, NC). Models predicting birth outcomes were fit using SAS General Linear Models Procedure (Proc GLM); covariates included environmental biomarker values, maternal race/ethnicity (white versus nonwhite), infant sex, and, except for those analyses predicting gestational age, gestational age. Models with $\Sigma$ DAPs included PON1 phenotype as tertiles or PON192 genotype $(R R, R Q, Q Q)$, or BuChE. OC models were adjusted for maternal age. Additional covariates were identified using bivariate analyses for biomarkers (Spearman correlation for continuous and $\chi^{2}$ or Mantel-Haenszel (M-H) for categorical variables with $p<0.15$ ), including those reported previously for OCs (7). Race, education, and marital status were highly correlated, and therefore only race was included in birth outcome models (3). Continuous biomarker values were log-transformed $\left(\log _{10}\right.$ to be comparable with references 5,20), and 0.5 was added to LDAPs to remove zero values. Potential covariates were added to the base model for each birth outcome, and backward stepwise elimination was used to remove covariates that altered biomarker betas by $<15 \%$. Final alkylphosphate models did not have entered any variables besides the base covariates. In OC models, the only covariate added to models was maternal pregnancy weight gain. However, OCs and birth outcomes are each known to be associated with both maternal weight gain and prepregnancy body mass index (BMI) (21-24). Therefore, to facilitate interpretation of the results, we present models adjusted for both variables and their interaction (using categorical variables for each, less than and equal to or greater than medians). The interaction term was not statistically significant, and using weight gain and BMI as continuous variables or quartiles did not change the DDE effect. Because pregnancy weight gain $(n=16)$ and lipids $(n=20)$ were missing for 194 women with DDE and PCB values, we constructed models in the subset of 178 with complete covariate data apart from lipids and report separately the models without and with lipid adjustment in the subset having all variables $(n=160)$. Using individual lipid-corrected values (i.e. ng/g lipid) did not change findings nor did adjusting for triglycerides and cholesterol instead of total lipids. Based on pharmacokinetics, the greatest OP exposure potential should exist if the slowest PON1 activity (first tertile or PON192RR genotype) were combined with high exposure (equal to or greater than median $\Sigma$ DAPs); the least potent subgroup would be fastest PON1 activity (third tertile or PON192 QQ genotype) with low exposure potential (less than median $\Sigma$ DAPs). Therefore, we compared least squares means for these extremes by examining the interactions between APs and PON1 or PON192 genotype for those models where a main effect at $p<0.1$ had been seen for urinary APs, PON1, or PON192.

\section{RESULTS}

Maternal biomarker levels are presented in Table 1. Maternal plasma DDE and PCB levels both had median values $<1$ $\mu \mathrm{g} / \mathrm{L}$. Median OC and OP concentrations were 1-100 $\mu \mathrm{g} / \mathrm{L}$, except for MDA, the malathion-specific metabolite that was detectable in only $20 \%$ of samples tested. $\Sigma$ DMP urinary metabolites were about threefold higher than $\Sigma$ DEP in urine. $\mathrm{PbB}$ levels were very low and not related to any covariates or birth outcomes.

Levels of OCs (DDE and PCB) were higher in older women, in white women, in married women, and those with college education (Table 2), suggesting an age-related association with higher socioeconomic status. DDE was higher in leaner women and lower in participants recruited after January 2001. 


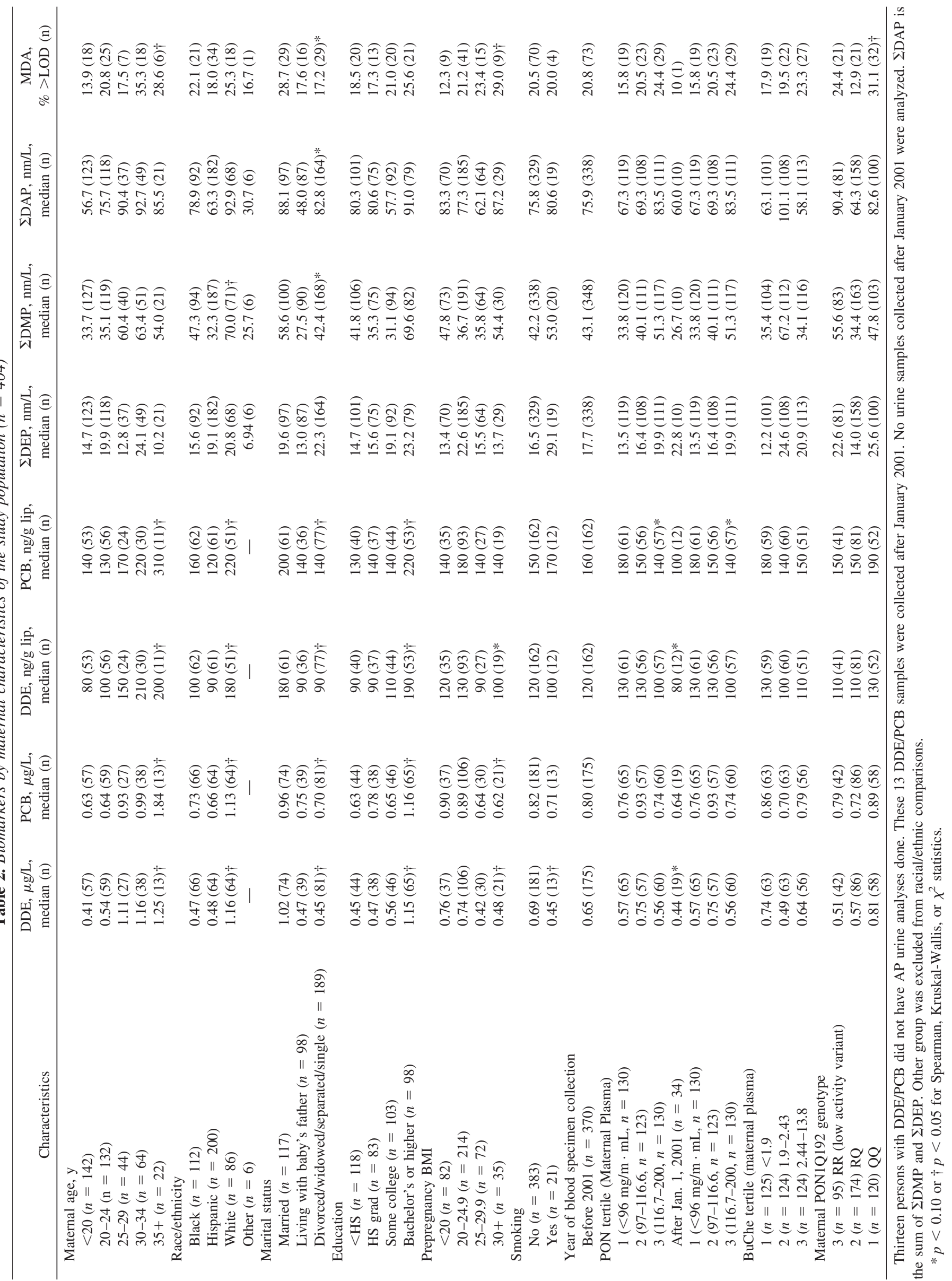




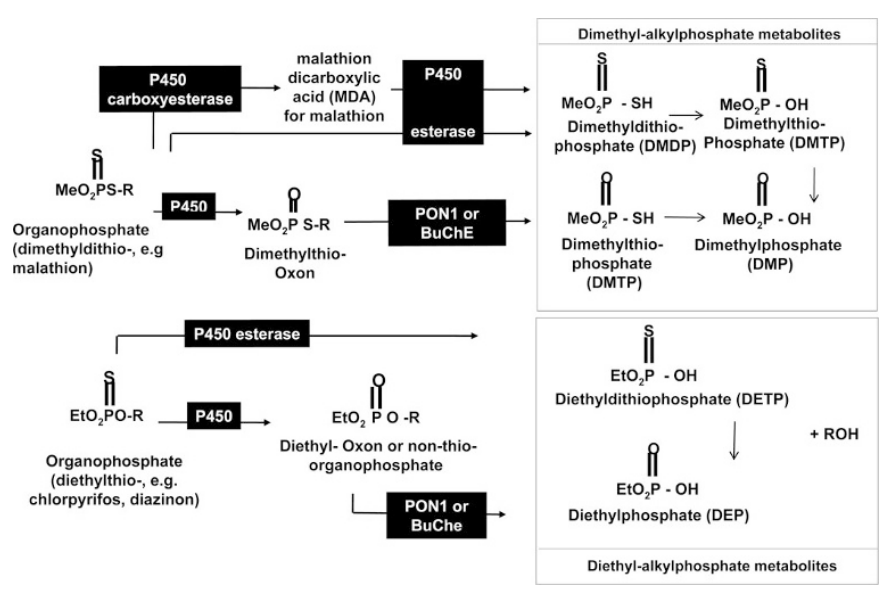

Figure 1. Roles of PON and BuChe in dimethyl- and diethyl-OP metabolism. PON1 and BuChe are esterases that cleave the RO-P moiety of OPs. BuChe, a suicide enzyme that binds dimethylphosphate, DMTP, DEP, or DMTP, is destroyed after activation, whereas PON1 is recycled. P-450 enzymes catalyze the first step of OP metabolism to an oxon, which PON1 or BuChe then de-esterifies at the RO- (ligand-ester) linkage, leading to an AP [dimethyl- top right and diethyl-phosphate (bottom right)]. ROH for the diethyl-OP chlorpyrifos is 2,5,6-trichloropyridinol. ROH is not shown for the dimethyl-OPs. APs may undergo further metabolism, including replacement of the thio-(S-) group with oxygen (O-), subsequent glucuronidation or sulfation by other enzymes, and excretion.
Urinary APs were significantly associated with two characteristics: race/ethnicity (¿DMPs) and marital status (¿DAPs). No meaningful trends with characteristics were seen for PON1, BuChe, or PON192 with $\Sigma$ DAPs or MDA (Table 2).

Relationships among urinary $\Sigma$ DAPs and MDA were explored in relation to possible metabolic pathways (Fig. 1). Between individual metabolites (data not shown), MDA was associated with the malathion metabolite DMTP (M-H = 6.61 , for MDA dichotomous $\geq$ or $<$ LOD versus tertiles of DMTP, $p=0.01, n=358)$ and with total $\Sigma$ DAPs (M-H 4.43, $p=0.035, n=348$ ). These associations were stronger when only samples with detectable APs were considered for MDA with DMDP $r_{\mathrm{S}} 0.64(n=23)$, with DMTP $\left(r_{\mathrm{S}} 0.46 n=70\right)$, with $\sum \operatorname{DMP}\left(r_{\mathrm{S}} 0.43 n=47\right)$, with $\Sigma \mathrm{DAP}\left(r_{\mathrm{S}} 0.38 n=72 ; p<0.05\right)$.

Multivariate models predicting birth outcomes from DDE, PCBs, and $\Sigma$ DAPs are shown in Table 3. Maternal DDE was inversely associated with birth weight and head circumference, although the relationship with birth weight was not statistically significant $(0.05<p<0.10$; adjusted for maternal age, race/ethnicity, BMI * weight gain interaction $(<$ and $\geq$ median), infant sex, and gestational age). Additional adjustment for lipids made little change in the estimates (Table 3). DDE was not a predictor of birth length, Ponderal index, or gestational age. PCBs were not significantly associated with any birth outcomes, although the estimate for gestational age suggested a 1-wk increase per $\log _{10}$ PCB $(p=0.07)$. Includ-

Table 3. Fetal growth and gestational age in relation to exposure biomarkers, Children's Environmental Health Study, Mount Sinai Hospital, 1998-2002

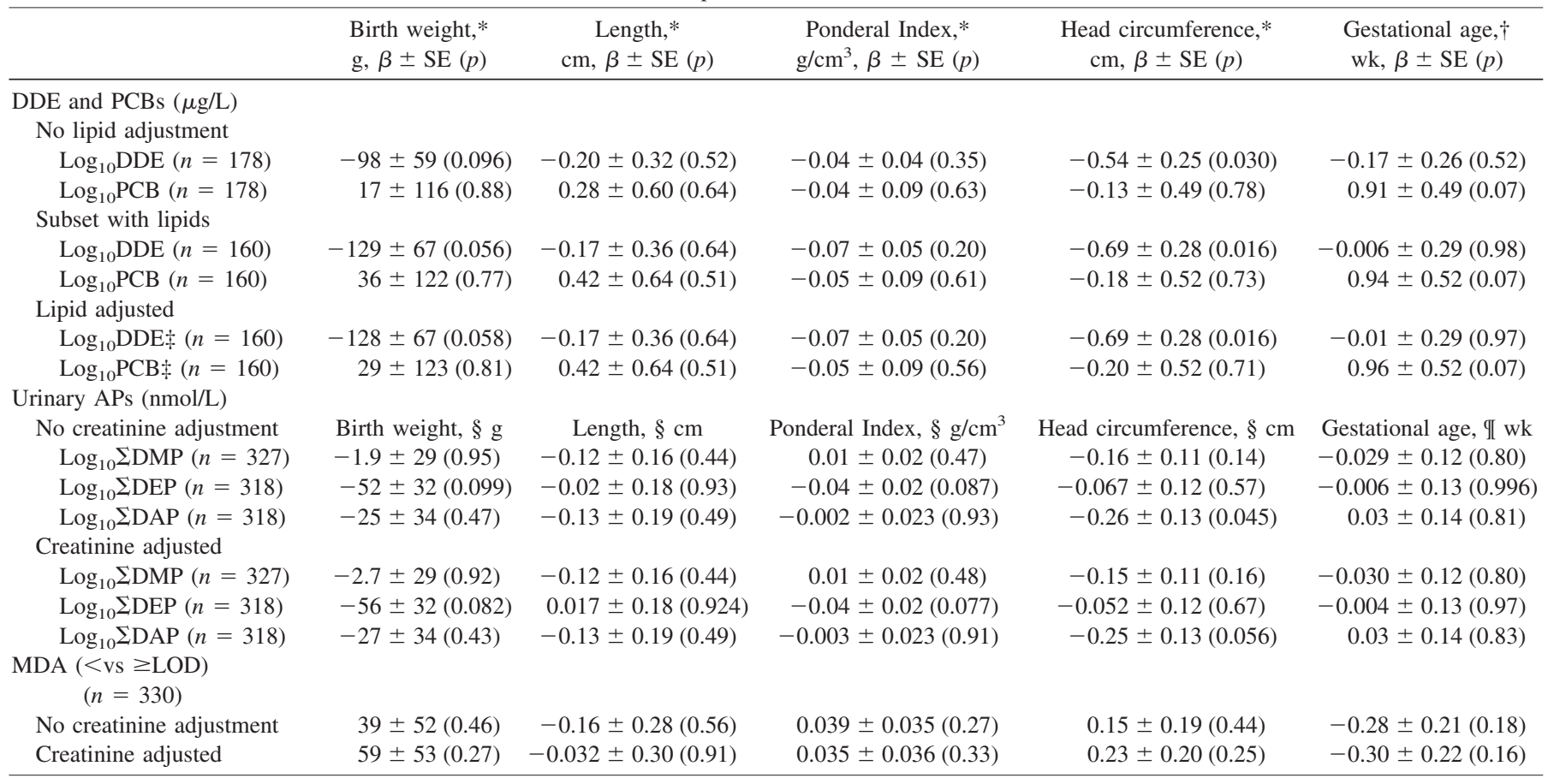

A value of 0.5 was added to the urinary APs before converting to logs. Urinary metabolite models excluded $25 \mathrm{samples}$ with $<20 \mathrm{mg} / \mathrm{dL} \mathrm{creatinine}$.

DDE and PCBs were done in a random subset of 192 women. Models are reported for the 178 with maternal weight gain and other covariates; of these, 160 had lipids measured. APs were done on samples collected before 2001.

* Adjusted for maternal age, race/ethnicity, maternal BMI* pregnancy weight gain (median quantiles), infant sex, and gestational age.

$\dagger$ Adjusted for maternal age, race/ethnicity, maternal BMI * pregnancy weight gain (median quantiles), and infant sex.

$\ddagger$ Adjusted additionally for maternal plasma lipids.

$\S$ Adjusted for race/ethnicity, maternal PON1 activity, infant gender, and gestational age.

II Adjusted for race/ethnicity, maternal PON1 activity, and infant gender. 
ing both PCBs and DDE in birth outcome models did not change effects of either compound.

The pronounced joint effect of both DDE levels and maternal weight gain on birth weight and head circumference prompted us to further explore these relationships while accounting for maternal prepregnancy BMI (Fig. 2). Lower maternal weight gain/BMI accompanied smaller birth weight or head circumference, higher DDE levels, and older maternal age. Thus, from high to low extremes of weight gain/BMI, birth weight decreased by $-270 \mathrm{~g}$ (crude or adjusted $\pm 84 \mathrm{SE}$, $p=0.0014, n=178)$ and head circumference decreased by $0.4-0.5 \mathrm{~cm}$ (crude or adjusted: $\pm 0.4 \mathrm{SE}, p=0.18$ ). Over the same extremes, DDE increased from 0.48 to $1.4 \mu \mathrm{g} / \mathrm{L}$ (unadjusted geometric means) and mean maternal age from 23 to $28 \mathrm{y}$ (Fig. 2). Therefore, DDE birth outcome models may have residual confounding due to our inability to disentangle these effects.

Maternal LDAPs exhibited weak, nonsignificant inverse associations with three birth outcomes: $\Sigma$ DEP with birth weight, $\Sigma$ DEP with Ponderal index, and $\Sigma$ DAP with head circumference (adjusted; Table 3). MDA was not associated with any birth outcomes $(p>0.15)$. Excluding or adjusting for mothers recruited after January 2001 did not change associations of APs with birth outcomes, although there were few such women in our sample. Creatinine adjustment made little further change in the estimates possibly because we had excluded the most dilute urine samples $(<20 \mathrm{mg} / \mathrm{dL}$ creatinine).

As reported previously (3), a strong association was observed between maternal PON1 activity and head circumference (Table 4). In models using tertiles instead of continuous PON1, head circumference was $0.62 \pm 0.18 \mathrm{~cm}$ smaller in the first (slow activity) compared with the third (fast activity) tertile of PON1 (adjusted, $n=382 ; p=0.0009$; not shown). Similarly, head circumference was $0.37 \pm 0.21 \mathrm{~cm}$ shorter for PON192RR (slow/slow allelotype, $n=95$ ) versus $P O N 192 Q Q$ (fast/fast, $n=120) ; p=0.082$, adjusted means; data not shown. Birth length was shorter by $0.68 \pm 0.30$ $\mathrm{cm}$ among mothers with $P O N 192 R R$ than PON192QQ allelotype $(p=0.026$, data not shown). The range of enzyme activities was similar for PON1 and BuChE (a ratio of 1.2-1.3 between the tertile cut points), but no associations were observed between exposures or birth outcomes with maternal BuChe.

Based on finding a main effect with APs $(p<0.1)$, we examined their interactions with PON1 and PON192 for birth weight ( $\Sigma \mathrm{DEP})$, head circumference ( $\Sigma \mathrm{DAP})$, and Ponderal index ( $\Sigma$ DEP). For birth length, which was associated with PON192, we examined interactions with $\Sigma$ DMP, SDEP, and $\Sigma$ DAP. This approach is consistent with the metabolic pathway of OP (Fig. 2) and our previous finding of an interaction between PON1 and 3,5,6- trichloropyridinol (3). For birth
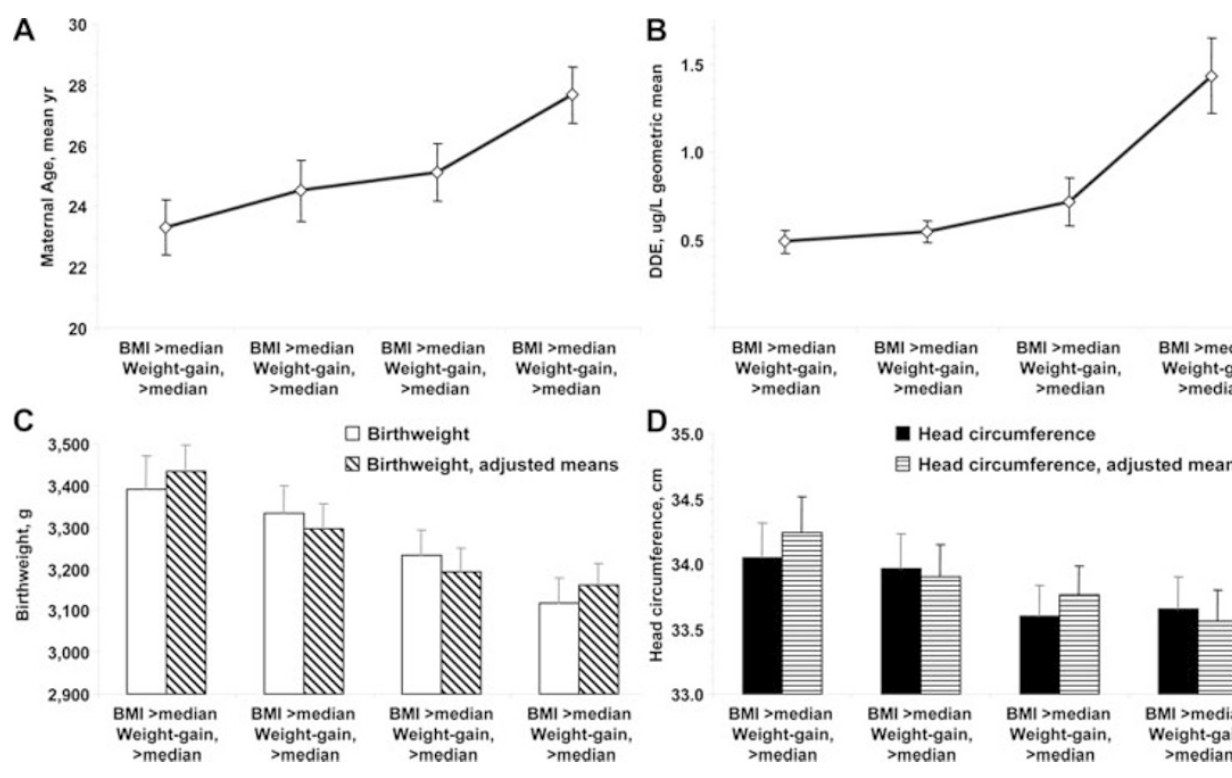

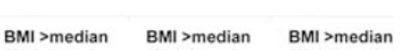
Weight-gain, Weight-gain, Weight-gain, >median >median >median

- Head circumference

目 Head circumference, adjusted means

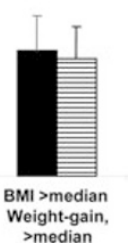

Figure 2. $(A, B)$ The plots show mean maternal age and geometric means of DDE by maternal pregnancy weight gain/BMI categories. $(C, D)$ The bar graphs show birth weight and head circumference means by weight gain/BMI, crude and adjusted for $\log _{10}$ DDE, maternal age, race/ethnicity, sex, and gestational age (models in Table 3). Error bars are SE.

Table 4. Regression coefficients ( \pm SE) of birth outcomes in relation to maternal PON1 and BuChe enzymatic activity, Children's Environmental Health Study, Mount Sinai Hospital, 1998-2002

\begin{tabular}{|c|c|c|c|c|}
\hline \multirow[b]{2}{*}{ Birth outcome } & \multicolumn{2}{|c|}{ PON1 enzymatic activity $(\mathrm{mg} / \mathrm{mL} \cdot \min )$} & \multicolumn{2}{|c|}{ BuChe enzymatic activity $(\mathrm{mg} / \mathrm{mL} \cdot \mathrm{min})$} \\
\hline & $\left(\beta^{*} 10^{3} \pm \mathrm{SE}^{*} 10^{3}\right)$ & $p$ & $\beta \pm \mathrm{SE}$ & $p$ \\
\hline Birth weight, ${ }^{*} \mathrm{~g}$ & $8.88 \pm 7.32$ & 0.226 & $31.8 \pm 330$ & 0.923 \\
\hline Birth length,* cm & $-0.004 \pm 0.04$ & 0.920 & $0.25 \pm 1.83$ & 0.889 \\
\hline Head circumference, $* \mathrm{~cm}$ & $0.081 \pm 0.028$ & 0.004 & $0.44 \pm 1.27$ & 0.728 \\
\hline Gestational age, $\dagger$ wk & $-0.033 \pm 0.029$ & 0.254 & $-0.45 \pm 1.32$ & 0.732 \\
\hline Ponderal Index* & $0.007 \pm 0.005$ & 0.135 & $0.024 \pm 0.23$ & 0.916 \\
\hline
\end{tabular}

The PON $\beta$ values are presented after being multiplied by $10^{3}$.

* Adjusted for sex, race, and gestational age.

$\dagger$ Adjusted for sex and race. 
Table 5. Adjusted means \pm SE of birth weight and length in relation to interactions between maternal urinary prenatal $\Sigma D A P$ levels, PON192 genotype, and PON1 activity, Children's Environmental Health Study, Mount Sinai Hospital, $1998-2002$

¿DEP and birth weight

\begin{tabular}{|c|c|c|c|c|c|}
\hline \multirow[b]{2}{*}{ Maternal PON1 tertile* } & \multicolumn{2}{|c|}{ Low maternal $\Sigma$ DEP $<$ median level } & \multicolumn{2}{|c|}{ High maternal $\Sigma$ DEP $\geq$ median level } & \multirow{2}{*}{$\begin{array}{l}p \text { value for } \Delta \text {-birth weight } \\
\text { by } \Sigma \text { DEP within PON1 }\end{array}$} \\
\hline & $n$ & Birth weight, g, mean \pm SE & $n$ & Birth weight, $g$, mean $\pm \mathrm{SE}$ & \\
\hline 1st (slow) & 60 & $3305 \pm 53$ & 53 & $3233 \pm 56 \dagger$ & 0.323 \\
\hline 2nd & 53 & $3348 \pm 57$ & 51 & $3282 \pm 57$ & 0.392 \\
\hline \multirow[t]{2}{*}{ 3rd (fast) } & 45 & $3396 \pm 64 \dagger$ & 56 & $3279 \pm 54$ & 0.138 \\
\hline & \multicolumn{2}{|c|}{ Low maternal $\Sigma$ DEP $<$ median level } & \multicolumn{2}{|c|}{ High maternal $\Sigma D E P \geq$ median level } & $p$ value for $\Delta$-birth weight \\
\hline Maternal PON192** & $\mathrm{n}$ & Birth weight, g,mean $\pm \mathrm{SE}$ & $\mathrm{n}$ & Birth weight, $g$, mean $\pm \mathrm{SE}$ & by $\Sigma$ DEP within genotype \\
\hline$R R$ (slow) & 39 & $3346 \pm 69$ & 55 & $3254 \pm 63 \ddagger$ & 0.291 \\
\hline$R Q$ & 84 & $3278 \pm 46$ & 66 & $3285 \pm 50$ & 0.907 \\
\hline$Q Q$ (fast) & 33 & $3453 \pm 60 \ddagger$ & 42 & $3232 \pm 52$ & 0.005 \\
\hline \multicolumn{6}{|c|}{$\Sigma$ DMP and birth length } \\
\hline & \multicolumn{2}{|c|}{ Low maternal $\Sigma$ DMP $<$ median level } & \multicolumn{2}{|c|}{ High maternal $\Sigma$ DMP $\geq$ median level } & \\
\hline Maternal PON1 tertile§ & $\mathrm{n}$ & Birth length, $\mathrm{cm}$, mean $\pm \mathrm{SE}$ & $\mathrm{n}$ & Birth length, $\mathrm{cm}$, mean $\pm \mathrm{SE}$ & $\begin{array}{l}p \text { value for } \Delta \text { length } \\
\text { by } \Sigma \text { DMP within PON1 }\end{array}$ \\
\hline 1 st (slow) & 61 & $51.1 \pm 0.3$ & 52 & $50.2 \pm 0.3$ & 0.032 \\
\hline 2nd & 55 & $50.3 \pm 0.3$ & 51 & $50.7 \pm 0.3$ & 0.258 \\
\hline \multirow[t]{2}{*}{ 3rd (fast) } & 49 & $50.4 \pm 0.3 \|$ & 58 & $50.8 \pm 0.3$ & 0.418 \\
\hline & \multicolumn{2}{|c|}{ Low $\Sigma$ DMP $<$ median level } & \multicolumn{2}{|c|}{ High $\Sigma$ DMP $\geq$ median level } & $n$ volue for 4 lenoth \\
\hline Maternal PON192凹 & $\mathrm{n}$ & Birth length, cm, mean $\pm \mathrm{SE}$ & $\mathrm{n}$ & Birth length, $\mathrm{cm}$, mean $\pm \mathrm{SE}$ & by $\Sigma$ DMP within genotype \\
\hline$R R$ (slow) & 43 & $50.6 \pm 0.4$ & 53 & $49.9 \pm 0.3 \neq \neq$ & 0.164 \\
\hline$R Q$ & 85 & $50.4 \pm 0.3$ & 70 & $50.7 \pm 0.3$ & 0.158 \\
\hline$Q Q$ (fast) & 35 & $51.0 \pm 0.3+\ddagger$ & 41 & $50.8 \pm 0.3$ & 0.695 \\
\hline
\end{tabular}

$* p$ value for the interaction term in the model $=0.878$.

$\dagger p$ value for extremes (third PON1/low $\Sigma$ DEP $v s$ first PON1/high $\Sigma$ DEP, $164 \mathrm{~g}$ ) $=0.042$.

** $p$ value for the interaction term in the model $=0.0755$.

$\$ p$ value for extremes (high PONQQ/low $\Sigma$ DEP $v s$ low activity PONRR/high $\Sigma$ DEP $199 \mathrm{~g}$ ) $=0.020$.

$\S p$ value for the interaction term in the model $=0.036$.

$\| p$ value for extremes (high PON1/low LDMP $v s$ low activity PON1/high $\Sigma$ DMP, $0.3 \mathrm{~cm}$ ) $=0.549$.

II $p$ value for the interaction term in the model $=0.230$.

$+\ddagger p$ value for highlighted extremes (high $P O N Q Q /$ low $\Sigma$ DMP $v s$ low activity $P O N R R /$ high $\Sigma$ DMP, $1.0 \mathrm{~cm}$ ) $=0.019$.

In the $\Sigma$ DMP-greater-than-the-median group, length was shorter among the $R R$ genotype than the $Q R(p=0.042)$ or $Q Q(p=0.041)$ genotypes.

The first tertile of PON1 and the RR PON192 genotype are the slower activity groups.

Means adjusted for race, sex, gestational age, and creatinine level. Urinary metabolites included samples with creatinine $>20 \mathrm{mg} / \mathrm{dL}$. Cutpoints for the PON1 second tertile were $>96$ to $<116.7 \mu \mathrm{g} / \mathrm{mL} / \mathrm{min}$.

weight, interaction terms were not significant for PON1$\sum$ DEP $(p=0.878)$ or PON192- $\sum$ DEP $(p=0.076)$ (Table 5). However, there was a 164-g deficit in birth weight between extremes of the interaction $(p=0.042)$, i.e. fast activityPON1 genotype/low $\Sigma$ DEP (least risk) versus slow-PON1/ high $\Sigma$ DEP (greatest risk). A similar effect between extremes was seen for the PON192* 2 DEP interaction $(-199, p=$ 0.020; Table 5). In addition, within only the fast $P O N 192 Q Q$ genotype, low $\Sigma$ DEP $* P O N 192 Q Q$ birth weight was higher than high $\Sigma \mathrm{DEP} *$ PON192QQ $(p=0.005$; Table 5). For birth length, the interaction was significant between $\Sigma$ DMP and PON1 but not PON192, yet effects were similar. Here, an exposure effect on birth length was seen within the slow activity PON1 tertile (first). Thus, mothers with slowest PON1 and high $\Sigma$ DMP had shorter babies $(-0.9 \mathrm{~cm})$ than low $\Sigma$ DMP ( $p=0.032$; Table 5). The effect was similar but not significant with slow PON192RR $(-0.6 \mathrm{~cm}$ in high $\Sigma$ DMP versus low $\Sigma$ DMP, $p=0.164)$. Moreover, birth length was significantly different between extremes of PON192 genotype with $\mathrm{EDMP}(-1.0 \mathrm{~cm}, p=0.019)$. Exposure did not modify the effect of PON1 or PON192 to reduce head circumference, nor were there any significant effects of alkylphosphates on the Ponderal index when interactions with PON1 were considered (data not shown).

\section{DISCUSSION}

Biomarker levels of DDE and PCBs, while low, are similar to those in other recent reports concerning women this age $(6,8,25)$. Our MDA and 2 DMP levels were higher than the recent CDC report (5\% $\geq$ LOD MDA, $20 \mathrm{~nm} / \mathrm{L}$ LDMP) but were half the levels reported among women working in agriculture in California (5). $\Sigma$ DEP levels in this population were similar to those in the National Health and Nutrition Examination Survey and in California women $(10 \mathrm{~nm} / \mathrm{L})$. ¿DMP levels were three times higher than $\Sigma$ DEP metabolites in our study, in the California study (5), and in national data for U.S. adult women in 1999-2000 (25). Both DDE and AP levels 
were lower among women recruited in later years (not age adjusted), as reported for OP pesticides in another New York City cohort of pregnant women (4). The strongest relationship among OP metabolites was MDA with DMDP, a primary malathion metabolite that is not produced via PON1. Substrates for both PON1 and BuChE are metabolites created by phase I type P-450 enzymes (Fig. 2), and therefore measurement of P-450 enzyme variants may be useful in addition to PON1 for assessing individual susceptibility to some OPs (Fig. 2). We did not measure these or other enzymes such as glucuronidase or sulfatase or additional OP-specific metabolites that might be formed through these pathways.

We observed smaller birth weight and head circumference in relation to higher maternal biomarker levels of DDE, although estimates were not statistically significant for birth weight. Positive weight gain and high maternal prepregnancy BMI are known to increase both birth weight and serum triglycerides (21) and also to decrease $\operatorname{DDE}(22,23)$ and $\mathrm{PCB}$ (24) levels. Therefore, the combined effect of maternal weight factors, DDE, and maternal lipids on birth outcomes is likely to be complex, and residual or unmeasured confounding among these variables may account for the observed associations, possibly further destabilized by our small sample size. For DDE, the effect size was in the range of 100 -g birth weight and $0.5-\mathrm{cm}$ head circumference accompanying a 10-fold change in DDE levels ( $1 \log _{10}$ unit). In addition, our exposure levels are even lower than other reports that did not find an effect of DDE on birth weight. Moreover, recent comprehensive reviews of this literature suggest that DDE effects on pregnancy, if any, are more likely to be related to pregnancy loss or preterm birth than to birth size $(26,27)$. Another possibility is that the DDE association with birth weight is related to unmeasured lipids, such as fatty acids, that covary with OCs (28). Adjustment for serum lipids did not change our DDE estimates as much as maternal pregnancy weight gain.

Birth weight was marginally affected by OP metabolite levels of $\Sigma$ DEP ( $<60 \mathrm{~g}$ lower birth weight per $\log _{10} \Sigma$ DEP). Birth length was affected by $\Sigma$ DMP, but only if maternal PON1 was considered. A small effect of $\Sigma$ DAP on head circumference was no longer present when the interaction of ¿DAP with PON1 was taken into account. In the California pregnancy study, inverse relationships were seen between ¿DMP and gestational age (5) but not with weight, length, Ponderal index, or head circumference. $\Sigma$ DEP levels were lower than $\Sigma$ DMP levels in our study, yet we saw an effect of $\Sigma$ DEP but not $\Sigma$ DMP on birth weight. It is possible that effects of $\Sigma$ DEP, if any, might be due to greater toxicity of the parent compounds or to different relative metabolism rates of OPs and their metabolites. Our findings are consistent with those in a minority population in northern Manhattan in which chlorpyrifos levels in cord plasma were associated with reduced birth weight and length (4). $\Sigma$ DEP includes nonspecific urinary metabolites of chlorpyrifos, but the proportionality between cord plasma and maternal urinary levels is not known.

Of interest, among several reported relationships of lowlevel exposures with birth outcomes, the effect sizes are similar (e.g. $-52 \mathrm{~g}$ in birth weight for $1 \log _{10}$ unit change in $\Sigma$ DEP [ $-22 \mathrm{~g}$ per $\log _{\mathrm{e}}$ $\Sigma$ DEP] in this study versus $-43 \mathrm{~g}$ for a $\log _{\mathrm{e}}$ unit change in blood chlorpyrifos in Whyatt et al. (4) and are $<100 \mathrm{~g} / \log _{\mathrm{e}}$ biomarker, similar in magnitude to studies having nonsignificant associations of DDE with birth weight $(20,29)$. In contrast, a larger and significant effect was found in a more highly exposed fish-eating population [-146 $\mathrm{g}$ birth weight/ln DDE (30)]. Finally, even a 200-g change in birth weight is unlikely to be clinically significant in a population of healthy infants such as ours, but it might be important among low birth weight infants. However, a 200 g-reduction is similar to that for smoking seven to eight cigarettes per day in the third trimester (31).

The influence of OPs on birth weight was stronger if individual susceptibility was taken into account. Individuals with lower PON1 activity are likely to have longer elimination rates and therefore higher OP levels in the body. Both phenotype and genotype are useful measures of susceptibility. Phenotype shows actual activity during pregnancy, but it is variable at any one time point (32), having weak associations with genotypes (15). In contrast, genotype may be considered an integrated indicator of PON1 activity; indeed, our suggestive trend of increasing birth weight with higher activity PON1 or PON192 in low $\Sigma$ DEP-exposed mothers is consistent with a previous report (33). The PONQ192R effect on head circumference is consistent with our previous report of lower PON1 and smaller head circumference (3). Here we see possible interactions of PON1 or PON192 and $\Sigma$ DEP levels with birth weight or $\Sigma$ DMP with birth length. These observations may explain some differences between our study and the California results; the two studies also had a different racial/ ethnic make up, which would alter PON1 genotype distribution and phenotype levels. Neither we nor the California study found an effect of $\mathrm{BuChE}$ on birth outcomes.

A strength of our study is the inclusion of prenatal exposure measurements in primiparous women. Our OC birth outcome findings may also represent a false-positive due to small numbers, as generally no effects have been observed even with quite large sample sizes $(26,27)$. A limitation of some biomarkers is potential misclassification of exposure reflected in a single measurement; this is especially true for urinary $\mathrm{LDAPs}$, which may not be highly reproducible between trimesters of pregnancy (10). The potential for coexposures to preformed OP metabolites in the environment exists, so these exposures may contribute to urinary $\Sigma$ DAPs levels. Although OC body burden (total amount of OC) is likely to change little during pregnancy (no elimination), measured concentrations can be reduced due to dilution by increased maternal weight. Therefore, our findings and those of similar studies between birth weight and pesticide biomarkers may reflect maternal factors other than chemical exposure, and it is possible that differences across studies can be clarified with better knowledge of total exposure and individual pharmacokinetic factors, including metabolic variation.

Acknowledgments. The authors thank the research team, including Stefanie Meisel, Martha Malagon, James Godbold, Karen Ireland, Zhisong Liu, and Robert Lapinski, for their valuable assistance. 


\section{REFERENCES}

1. Eskenazi B, Bradman A, Castorina R 1999 Exposures of children to organophosphate pesticides and their potential adverse health effects. Environ Health Perspect 107:409-419

2. Schantz SL, Widholm JJ, Rice DC 2003 Effects of PCB exposure on neuropsychological function in children. Environ Health Perspect 111:357-576

3. Berkowitz GS, Wetmur JG, Birman-Deych E, Obel J, Lapinski RH, Godbold JH, Holzman IR, Wolff MS 2004 In utero pesticide exposure, maternal paraoxonase activity, and head circumference. Environ Health Perspect 112:388-391

4. Whyatt RM, Rauh V, Barr DB, Camann DE, Andrews HF, Garfinkel R, Hoepner LA, Diaz D, Dietrich J, Reyes A, Tang D, Kinney PL, Perera FP 2004 Prenatal insecticide exposures and birth weight and length among an urban minority cohort. Environ Health Perspect 112:1125-1132

5. Eskenazi B, Harley K, Bradman A, Weltzien E, Jewell NP, Barr DB, Furlong CE, Holland NT 2004 Association of in utero organophosphate pesticide exposure and fetal growth and length of gestation in an agricultural population. Environ Health Perspect 112:1116-1124

6. Fitzgerald EF, Hwang SA, Langguth K, Cayo M, Yang BZ, Bush B, Worswick P, Lauzon T 2004 Fish consumption and other environmental exposures and their associations with serum PCB concentrations among Mohawk women at Akwesasne. Environ Res 94:160-170

7. Wolff MS, Deych E, Ojo F, Berkowitz GS 2005 Predictors of organochlorines in New York City pregnant women, 1998-2001. Environ Res 97:170-177

8. Wolff MS, Teitelbaum SL, Lioy PJ, Santella RM, Wang RY, Jones RL, Caldwell KL, Sjodin A, Turner WE, Li W, Georgopoulos P, Berkowitz GS 2005 Exposures among pregnant women near the World Trade Center site on 11 September 2001. Environ Health Perspect 113:739-748

9. U.S. Environmental Protection Agency (EPA) 2001 Organophosphate pesticides in food: A primer on reassessment of residue limits. Office of Pesticide Programs. Available at: www.epa.gov/pesticides/op/primer.htm

10. Bradman A, Eskenazi B, Barr DB, Bravo R, Castorina R, Chevrier J, Kogut K, Harnly ME, McKone TE 2005 Organophosphate urinary metabolite levels during pregnancy and after delivery in women living in an agricultural community. Environ Health Perspect 113:1802-1807

11. Berkowitz GS, Obel J, Deych E, Lapinski R, Godbold J, Liu Z, Landrigan PJ, Wolff MS 2003 Exposure to indoor pesticides during pregnancy in a multiethnic, urban cohort. Environ Health Perspect 111:79-84

12. Barr DB, Allen R, Olsson AO, Bravo R, Caltabiano LM, Montesano A, Nguyen J, Udunka S, Walden D, Walker RD, Weerasekera G, Whitehead RD Jr, Schober SE, Needham LL 2005 Concentrations of selective metabolites of organophosphorus pesticides in the United States population. Environ Res 99:314-326

13. Bravo R, Caltabiano LM, Weerasekera G, Whitehead RD, Fernandez C, Needham LL, Bradman A, Barr DB 2004 Measurement of dialkyl phosphate metabolites of organophosphorus pesticides in human urine using lyophilization with gas chromatography-tandem mass spectrometry and isotope dilution quantification. J Expo Anal Environ Epidemiol 14:249-259

14. Parsons PJ 1992 Monitoring human exposure to lead: an assessment of current laboratory performance for the determination of blood lead. Environ Res 57:149162

15. Chen J, Kumar M, Chan W, Berkowitz G, Wetmur JG 2003 Increased influence of genetic variation on PON1 activity in neonates. Environ Health Perspect 111:14031409
16. Ellman GL, Courtney KD, Andres V Jr, Feather-Stone RM 1961 A new and rapid colorimetric determination of acetylcholinesterase activity. Biochem Pharmacol 7:88-95

17. Chen J, Germer S, Higuchi R, Berkowitz G, Godbold J, Wetmur JG 2002 Kinetic polymerase chain reaction on pooled DNA: a high-throughput, high-efficiency alternative in genetic epidemiological studies. Cancer Epidemiol Biomarkers Prev 11:131-136

18. Wetmur JG, Kumar M, Zhang L, Palomeque C, Wallenstein S, Chen J 2005 Molecular haplotyping by linking emulsion PCR: analysis of paraoxonase 1 haplotypes and phenotypes. Nucleic Acids Res 33:2615-2619

19. Phillips DL, Pirkle JL, Burse VW, Bernert JT Jr, Henderson LO, Needham LL 1989 Chlorinated hydrocarbon levels in human serum: effects of fasting and feeding. Arch Environ Contam Toxicol 18:495-500

20. Farhang L, Weintraub JM, Petreas M, Eskenazi B, Bhatia R 2005 Association of DDT and DDE with birth weight and length of gestation in the Child Health and Development Studies, 1959-1967. Am J Epidemiol 162:717-725

21. Di Cianni G, Miccoli R, Volpe L, Lencioni C, Ghio A, Giovannitti MG, Cuccuru I, Pellegrini G, Chatzianagnostou K, Boldrini A, Del Prato S 2005 Maternal triglyceride levels and newborn weight in pregnant women with normal glucose tolerance. Diabet Med 22:21-25

22. Wolff MS, Britton JA, Teitelbaum SL, Eng S, Deych E, Ireland K, Liu Z, Neugut AI, Santella RM, Gammon MD 2005 Improving organochlorine biomarker models for cancer research. Cancer Epidemiol Biomarkers Prev 14:2224-2236

23. Perry MJ, Ouyang F, Korrick S, Venners SA, Altshul L, Xu X, Wang X 2005 Body mass index and serum 1,1,1-trichloro-2,2-bis(p-chlorophenyl)ethane in nulliparous Chinese women. Cancer Epidemiol Biomarkers Prev 14:2433-2438

24. Stewart P, Reihman J, Lonky E, Darvill T, Pagano J 2000 Prenatal PCB exposure and Neonatal Behavioral Assessment Scale (NBAS) performance. Neurotoxicol Teratol 22:21-29

25. CDC 2005 National Report on Human Exposure to Environmental Chemicals Available at: http://www.cdc.gov/exposurereport/.

26. Longnecker MP 2005 Invited commentary: why DDT matters now. Am J Epidemiol 162:726-728

27. Rogan WJ, Chen A 2005 Health risks and benefits of bis(4-chlorophenyl)-1,1,1trichloroethane (DDT). Lancet 366:763-773

28. Grandjean P, Bjerve KS, Weihe P, Steuerwald U 2001 Birthweight in a fishing community: significance of essential fatty acids and marine food contaminants. Int J Epidemiol 30:1272-1278

29. Fenster L, Eskenazi B, Anderson M, Bradman A, Harley K, Hernandez H, Hubbard A, Barr DB 2006 Association of in utero organochlorine pesticide exposure and fetal growth and length of gestation in an agricultural population. Environ Health Perspect 114:597-602

30. Weisskopf MG, Anderson HA, Hanrahan LP, Kanarek MS, Falk CM, Steenport DM Draheim LA 2005 Maternal exposure to Great Lakes sport-caught fish and dichlorodiphenyl dichloroethylene, but not polychlorinated biphenyls, is associated with reduced birth weight. Environ Res 97:149-162

31. Bernstein IM, Mongeon JA, Badger GJ, Solomon L, Heil SH, Higgins ST 2005 Maternal smoking and its association with birth weight. Obstet Gynecol 106:986991

32. Costa LG, Vitalone A, Cole TB, Furlong CE 2005 Modulation of paraoxonase (PON1) activity. Biochem Pharmacol 69:541-550

33. Chen D, Hu Y, Chen C, Yang F, Fang Z, Wang L, Li J 2004 Polymorphisms of the paraoxonase gene and risk of preterm delivery. Epidemiology 15:466-470 